\title{
BMJ Open Integrated decision-making model for community-based rehabilitation service utilisation among persons with severe mental illness in China: protocol for a cross-sectional, mixed-methods study
}

\author{
Ruoxi Wang, ${ }^{1}$ Shangfeng Tang, ${ }^{1}$ lan Shaw, ${ }^{2}$ Zhanchun Feng, ${ }^{1}$ Zhuo Chen, ${ }^{3}$ \\ Yuxiong Luo, ${ }^{4}$ Hongxun Song, ${ }^{1}$ Tailai Wu, ${ }^{1}$ Qian Fu, ${ }^{1}$ Hang Fu, ${ }^{1}$ Yueying Huang, \\ Xiaoyu Chen, ${ }^{1}$ Da Feng ${ }^{5}$
}

To cite: Wang $\mathrm{R}$, Tang $\mathrm{S}$, Shaw I, et al. Integrated decision-making model for community-based rehabilitation service utilisation among persons with severe mental illness in China: protocol for a cross-sectional, mixedmethods study. BMJ Open 2018;8:e21528. doi:10.1136/ bmjopen-2018-021528

- Prepublication history for this paper is available online. To view these files, please visit the journal online (http://dx.doi. org/10.1136/bmjopen-2018021528).

Received 15 January 2018 Revised 21 September 2018 Accepted 31 October 2018
Check for updates

\section{(c) Author(s) (or their} employer(s)) 2018. Re-use permitted under CC BY-NC. No commercial re-use. See rights and permissions. Published by BMJ.

For numbered affiliations see end of article.

Correspondence to

Dr Da Feng; fdnunu@163.com

\section{ABSTRACT}

Introduction A common problem low-income and middleincome countries face is the scarcity of communitybased rehabilitation (CBR) resources and low service utilisation among persons with severe mental illness (SMI). Despite this problem, the factors and pathways followed influencing one's decision on service utilisation in China have not been fully comprehended. This study aims to develop a theory-based model that systematically describes the integrated decision-making process of mental health CBR utilisation among persons with SMI in China.

Methods/Design This cross-sectional, mixed-methods study involves three main stages and is expected to last 3 years, from January 2018 to December 2020. In stage 1, the Social Exchange Theory is deployed as an analytical framework to comprehensively capture factors associated with tendency to use CBR services in China using semistructured interview methodology involving patients with SMI, their primary caregivers and CBR service providers. In stage 2, interpretive structural modelling will be applied to analyse the relationships between factors in different dimensions, at different levels and with different levels of impact. Stage 3 involves a multiregion survey among at least 300 family decision-makers (either the patient or their caregivers) in six communities in three cities to statistically validate the initial model derived in stage 2 using a further structural equation modelling.

Ethics and dissemination Ethical approval was granted by the Ethics Committee of Tongji Medical College, Huazhong University of Science and Technology (No 2017S319). All interviewees will be provided with written information about the study, and a signed consent will be retrieved prior to the interview. Rules on confidentiality and anonymity of data will be strictly followed. The findings of this study will be disseminated via international and domestic peer-reviewed journals, reports, conference presentations and symposium discussions. Reports will be submitted to the National Natural Science Foundation of China.
Strengths and limitations of this study

- This study is one of the few studies that tend to provide a systematic understanding of the determinants and decision-making process of the utilisation of mental health community-based rehabilitation (CBR) services among patients with severe mental illnesses in low-income and middle-income countries (LMICS).

- This study is designed based on the Social Exchange Theory, a model widely used in the social science field, to systematically identify the driving factors from various layers and clusters.

- This study employs structural equation modelling (SEM) to quantitatively determine the impact the underlying factors (such as stigma, subjective norm, Chinese cultural value, and trust between the provider and the user) pose on CBR service utilisation.

- Given the diversity of healthcare systems, caution must be taken in applying findings to rural China or other LMICs.

- Although the study aims to investigate associations, identification of causal relationships will of course have to await further investigation and validation using methods such as a randomised controlled trial.

\section{INTRODUCTION}

The past few decades have witnessed a sharp increase in the global challenge posed by mental illness. The threefold challenge involves the large and still increasing absolute need for mental healthcare, ${ }^{12}$ especially for people with severe mental illnesses $(\mathrm{SMI})^{34}$; the absolute lack of professional resources; and significant variation in the distribution of the limited professional resources between low-income and high-income countries. ${ }^{56}$ This, therefore, leaves nearly $75 \%-85 \%$ of people in low-income and middle-income countries (LMICs) with SMI untreated, ${ }^{7}$ bringing about 
high caregiving burden to their family and the society. In response to such critical situation, programmes such as the mental health Gap Action Programme (mhGAP) and the Movement for Global Mental Health have been initiated with an aim to scale up the coverage of mental healthcare in LMICs. ${ }^{89}$ The community-based rehabilitation (CBR) model has been repeatedly recommended as a valid means to provide affordable, accessible and effective services to meet a wide range of needs of persons with chronic SMI. ${ }^{10} 11$

China, a middle-income country accounting for $18 \%$ of the world population and $17 \%$ of the global disease burden of psychiatric-related illness, ${ }^{12}$ is also encountering a significant gap between need and supply in mental health services, and therefore the potential disrupt and burden that patients with untreated SMI pose to their family and community. ${ }^{13}$ In accordance with the WHO recommendations, the Chinese government has issued several national-level policies to establish a nationwide mental health CBR network for patients with SMI since 2008. ${ }^{14-16}$ Since 2009, community-based physical rehabilitative services, including follow-ups, health education and physical examination, have been part of the National Essential Public Health Service Package and are regularly provided to every patient with SMI by community healthcare centres (CHCs) free of charge. The National Mental Health Law, ${ }^{17}$ launched in 2013, provides further instructions to build a closer hospital-CHC collaboration to deliver better mental healthcare services.

Meanwhile, most CHCs in China are providing the aforementioned physical rehabilitative services to patients with stable or no symptoms in the community under the supervision of a psychiatric hospital or the department of psychiatry from a general hospital. In this collaborative network, general practitioners or nurses at CHCs are mainly responsible for providing suggestions regarding medication and daily caring, monitoring symptoms, assessing the severity of illness and referring the patient to a superior hospital when they meet admission criteria. Besides providing inpatient care, psychiatric professionals at secondary or tertiary hospitals issue prescriptions for patients at outpatient clinics and provide technical guidance and training to healthcare workers from CHCs. Some better-off cities, such as Beijing, Shanghai, Shenzhen and Wuhan, have recently borrowed from Western experience and practice to incorporate the somatic healthcare-based CBR network with psychological and social services to meet different types of needs (eg, psychological and functional needs). ${ }^{18}$ In these cities, psychiatric rehabilitation institutes have been built in the community, mostly near or even in the CHCs, and can provide psychological and functional rehabilitation services to patients who live in the community. ${ }^{192}$

However, similar to the situation in other LMICs, ${ }^{21}$ CBR programmes in China tend to confront various complex situations on the ground. One of the frequently occurring and critical problems is the low utilisation of CBR services: a large proportion of patients have discontinued treatments for various reasons, leaving only $37.1 \%$ of patients with SMI who have regularly used mental health services, ${ }^{22}$ and a lot fewer who have positively sought social services. ${ }^{2023}$ This has resulted in a waste of resources, even in a context where a huge gap exists between a large amount of absolute need and scarce professional resources. ${ }^{23}{ }^{24}$ Approaches such as increasing the supplyside resource allocation and promoting the demand-side to make full use of the available resources have been considered as potential means to address this problem. However, it has also been noted that the former requires long policy-making process and may not achieve effective outcomes if the demand-side lacks awareness or willingness to use CBR services. Meanwhile, the latter has been regarded with higher feasibility, and therefore worth special attention. ${ }^{2425}$ This requires comprehensive understanding of how the driving factors and the underlying pathways coinfluence one's tendency to use CBR services.

\section{KNOWLEDGE GAPS TO BE ADDRESSED}

Investigation of one's willingness to use healthcare services starts from the investigation of one's help-seeking behaviour and general understanding of mental illness. Compared with the large number of studies in the physical health field, fewer studies focused on investigating one's decision on the utilisation of mental health services.

\section{Lack of comprehensive understanding of the driving factors for seeking mental health services}

Of the small number of studies that examined the determinants of mental health service utilisation, a large proportion looked into the predictors of peoples' intention to use mental health services. These studies have revealed three main findings.

First, decisions are influenced by a wide range of factors, among which some are 'forces' with a direction of impact. For instance, structural barriers, such as poor access to professional service and low income,${ }^{26}$ significantly impede one's service utilisation. Due to wrong attribution of cause or beliefs about mental illness (eg, some people with Asian worldviews tend to attribute the cause to personal failure or interpersonal problems, whereas some Chinese are more likely to attribute the cause of mental health problems to somatic disorders, rather than psychological/psychiatric problems), one may merely perceive the helpfulness of psychiatric treatments. ${ }^{27} 28$ Self-stigma refers to impaired self-esteem or feelings of shame when persons with mental illness internalise stigmatising attitudes, such as prejudice and discrimination, held by the public ('public stigma'). ${ }^{29}$ These feelings predict a less positive view towards service utilisation, although one can feel the need for care. ${ }^{30}$ Nevertheless, positive past experience, ${ }^{29}$ high sense of perceived need ${ }^{31}$ and social support ${ }^{32}$ may increase the likelihood of service utilisation.

Second, besides observable factors such as educational level, there are some essential underlying factors deeply 
embedded in the political, socioeconomic and cultural context of the Chinese society. ${ }^{33} 34$ These factors include public and self-stigma, ${ }^{35} 36$ subjective norm, ${ }^{37}$ attitude from significant others ${ }^{38} 39$ and so on. For instance, a positive relationship between public stigma and self-stigma ${ }^{40}$ and the positive effect of government's mass education on reducing stigma ${ }^{41}$ has been found. Compared with individualism societies, interpersonal relatedness is stronger in collectivistic societies, and the subjective norm plays a more important role in one's decision-making towards a behaviour. ${ }^{37}$ In Chinese societies, collectivist and family-oriented culture is greatly valued, and therefore the decision on whether to use CBR services lies not only on the patient themselves, rather on a consensus achieved among family members, especially between the patient and their family caregivers. ${ }^{37}{ }^{42}$ The decision-making becomes complex because of the number of factors involved.

Third, factors from different layers and directions may interact with each other, and therefore jointly affect one's decision. ${ }^{43}$ For instance, the absence of significant relationship between symptom severity and help-seeking intention can be attributed to the negative net force between stigma and actual service need. ${ }^{44}$

Meanwhile, knowledge in this field is mostly based on studies conducted in high-income countries ${ }^{45}{ }^{46}$ leaving China and its cultural or service-related factors underinvestigated. ${ }^{47}$ The limited number of studies that have focused on Chinese population were mainly carried out by scholars from Taiwan and Hong Kong, and the attention has been paid to investigating the public's understanding towards mental health service utilisation rather than the perspectives of persons with SMI. On the one hand, these studies have explored the influencing mechanisms of underlying factors, such as Chinese cultural beliefs, ${ }^{42}$ subjective norm and attribution of the cause of illness, ${ }^{37}$ on the decision-making process. On the other hand, they have also revealed the social and cultural heterogeneity within the ethnic Chinese population, ${ }^{48}$ which calls for context-driven studies considering the culturally rooted determinants of mental health service utilisation in mainland China. Furthermore, compared with the studies investigating the barriers (eg, stigma, lack of access to care, insufficient income and so on), less attention has been paid to identifying the facilitators (eg, high level of health literacy, sufficient social support and so on),$^{32}$ especially those from the provider side (eg, mediabased or other user-friendly service delivery strategies, ${ }^{49}$ high-quality services, trust in the provider, ${ }^{32}$ and so on). Although a positive relationship between higher quality services and stronger willingness to use CBR services has been documented, ${ }^{44}{ }^{50}$ the underlying mechanism has not been fully comprehended. In consideration of the complexity of factors, the largely underinvestigated underlying mechanisms and the under-represented population, an advanced theory-based model is needed to understand the decision-making process in mainland Chinese families (service users) on whether to use CBR services.
Lack of sophisticated models to explain the drivers of seeking mental health CBR services

Studies that employ theory-based models to systematically understand the determinants of service utilisation remain scarce in this field. ${ }^{37}$ Some models, such as the Andersen and Aday's Health Services Utilisation Model $^{51}$ and the Health Belief Model, ${ }^{52}$ have been introduced into this field to offer a good overview of the decision-making process. Andersen and Aday's mode ${ }^{53}$ indeed contributes to the classification of predictors by classifying them into three main groups, namely 'Need' (refers to perceived need for services, such as severity, chronicity, frequency of onset and so on), 'Predisposing factors' (age, gender, level of education and so on) and 'Enabling factors' (insurance, income and so on). It also outlines several underlying/macro-sociological factors, including political (health policy and so on), geographical (physician population ratio and so on) and environmental (population health indices) factors. ${ }^{53} 54$ Nevertheless, it fails to delineate the interaction of factors from different layers or dimensions. ${ }^{5155}$ Various studies have also questioned its explanatory power in the mental health field by pointing out the limited proportion of variance related to service utilisation it accounts for. ${ }^{565}$

The Health Belief Model conceptualises factors influencing one's help-seeking behaviour into five main constructs, namely 'Perceived Benefits', 'Perceived Barriers', 'Perceived Susceptibility', 'Perceived Severity' and 'General Health Motivation'. Since being introduced to the mental health field, analyses consistently suggest 'Perceived Benefits' and 'Perceived Barriers' to be direct predictors of help-seeking behaviours. ${ }^{58} 59$ Nevertheless, this theory has also been criticised for lack of validity since several studies revealed one or more core constructs of the model (such as 'Perceived Susceptibility' and 'General Health Motivation') to be not significant. O'Connor et $a \tilde{l}^{2}$ even considered this theory, as a whole, of limited explanatory power (as it overall explains $25 \%$ of the variance).

Due to the lack of validity of these two models, an alternative model is needed to better categorise the influencing factors, and therefore clearly explain the pathways these factors influence each other.

\section{Potentially feasible model}

The Social Exchange Theory (SET) is a social psychological and sociological perspective that explains social change and stability as a process of negotiated exchange between parties. The theory proposes that individuals are motivated to conduct an activity if they feel the benefits are more rewarding than the cost of it. ${ }^{60}$ To be specific, it illustrates the relationships within and between three core elements (costs, rewards and outcomes) involved in one's decision-making process: one's attitude towards an action depends on whether the motivation (accumulation of various rewards) overcomes the obstacles (accumulation of various costs); the worth of continuing the action is judged based on the outcome (actual costs 
paid and actual rewards gained) in the last action. In other words, this assumption conforms to previous findings regarding the direct relationship between decision-making, 'Perceived Costs' and 'Perceived Benefits' in the mental health field. ${ }^{58}{ }^{59}$ Additionally, the SET allows coexistence of factors from multiple layers, which leaves space for further pathway analysis of the driving factors. In this case, we regard the SET with potential to serve as a theoretical framework to delineate one's decision-making process.

To the best of our knowledge, only two studies have been retrieved using this theory to explain one's decision on mental health service utilisation: in Hamrin $e t a r^{61}$ literature review, the SET was adopted as the theoretical framework to guide qualitative identification of the rewards and costs that influence parents' decision-making on the utilisation of paediatric psychotropic medication services; also under the SET framework, Prizer et $a l^{62}$ revealed the direct association between neurologists' perceived costs and benefits of palliative care and their reported referral practice for their patients with Parkinson's disease. However, the interfactor relationships have been underexplored in either of these two studies.

Structural equation modelling (SEM) is a mathematical modelling technique that includes two basic models: measurement model and structural model. The former allows quantitative measurement of a latent, or in other words an underlying, factor by invoking observed variables, whereas the latter imputes the relationship between latent variables. Aided with SEM, various scholars have used the SET to explore the interactive pathways (accumulation or counteraction) among driving factors from multiple layers to explain one's decision-making process in the business management field. ${ }^{634}$

\section{AIM AND OBJECTIVES OF THE STUDY}

Our aim with this cross-sectional, mixed-methods study is to develop an effective decision-making model based on the SET to systematically capture the factors influencing the family integrated choice on the use of mental health
CBR services in China's context. We refer 'family integrated choice' to the final decision a family with patients with SMI reaches by considering a wide range of factors, including perspectives from other significant others (family members).

To achieve the aim of this study, we have two objectives:

- Comprehensively identify the major barriers and facilitators affecting family's integrated choice (involving patients with SMI and their family caregivers), with special attention paid to the factors rooted in the context of an LMIC and those from the provider side.

- Explain the complex interactive relationships among factors from multiple layers and dimensions to identify factors of intervention.

\section{THEORETICAL FRAMEWORK}

This study will therefore employ the SET as the theoretical framework and incorporate the 'need' and 'predisposing factors' in Andersen and Aday's model to combine their strengths and to maximise its explanatory power.

In the theoretical framework (figure 1), factors are classified into four clusters: (1) predisposing factors (including personal sociodemographic characteristics), (2) motivation (including perceived needs, encouragement from significant others and so on), (3) rewards (including perceived/expected benefits such as symptom relief, self-efficacy, positive interpersonal relationship and so on) and (4) costs (including attitudinal and structural obstacles such as self-stigma, poor access to care, traditional Chinese beliefs about the cause of illness and so on). Within each cluster, factors can be further classified as observable (micro-level) and underlying (meso-level/ macro-level factors), and the impact accumulates from fundamental factors to observable factors. For instance, one's unperceived need for CBR service is one of the observable factors in the motivation cluster, and it can be influenced by their wrong beliefs about the cause of illness, ${ }^{48}$ which is an underlying factor. One's causal attribution may be affected by Asian cultural values such as mind-body holism and the latter is a macro-level/fundamental-level factor. ${ }^{27}$ In other words, influenced by the

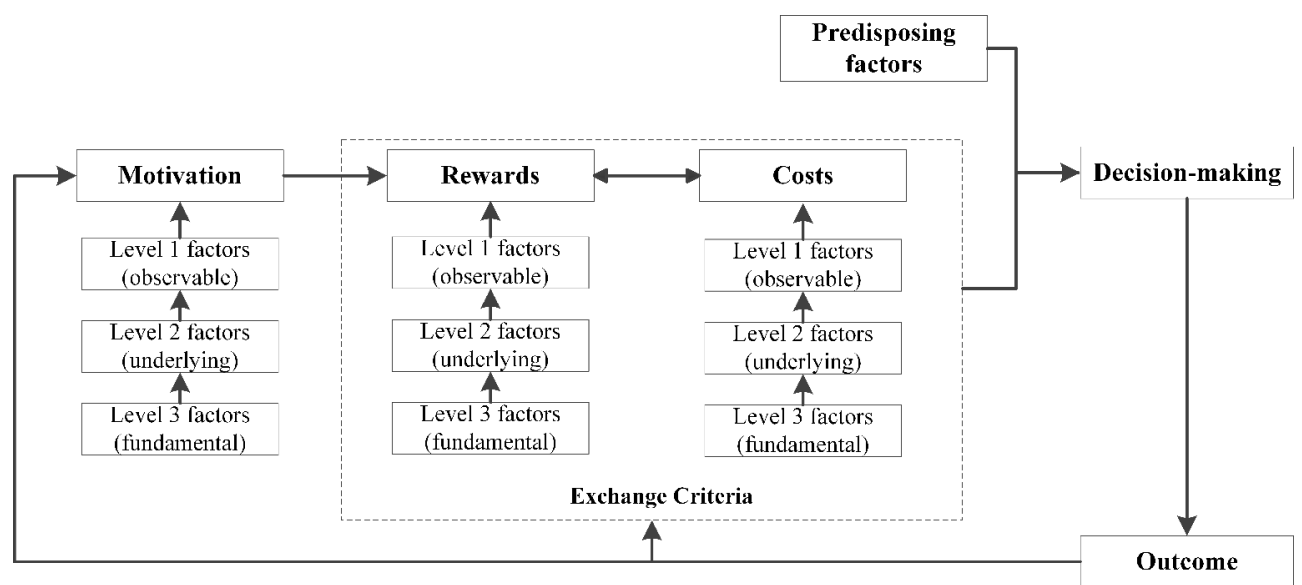

Figure 1 Decision-making model on the utilisation of community-based rehabilitation services among persons with severe mental illness. 
mind-body holism value, compared with Caucasian, Chinese are more likely to attribute the cause of mental health problems to somatic disorders, and therefore tend to perceive higher needs for physical care rather than psychiatric care. This may result in less likelihood of mental health service utilisation in Chinese population. Between clusters, there is a trade between rewards and costs. The net force of these four clusters of factors determines the integrated decision on whether to use CBR services. The actual rewards and costs will then affect the value of each cluster in the next decision-making process.

\section{RESEARCH DESIGN}

In consideration of the variety and complexity of the on-the-ground factors, this study uses a mixed-methods approach (qualitative and quantitative) conducted in three stages between January 2018 and December 2020:

- Stage 1: preidentify relevant factors under the theoretical framework.

- Stage 2: form the initial (theoretical) model using interpretive structural modelling (ISM).

- Stage 3: statistically validate the theoretical model using SEM.

\section{Stage 1: preidentify relevant factors under the theoretical framework}

Face-to-face semistructured interviews will be used to capture potential factors influencing the decision-making on CBR service utilisation. The grounded theory methodology (GTM) paradigm will be employed for data collection and analysis in order to extract all information, classify/summarise potential factors and sort out the relationships among these factors, and therefore preliminarily depict how these factors influence one's tendency to use CBR services.

\section{Participant recruitment}

Taking into consideration the Chinese culture that the decision on whether to use CBR services is usually a consensus achieved between the patient and their primary caregiver, patients and their family primary caregivers will both be interviewed. Health workers and social workers involved in the provision of CBR services are considered as key informants and will be interviewed.

We categorise patients with SMI into four groups (table 1). Three registered families in each of these four groups will be selected, and the patients and their family caregivers will be interviewed. In consideration of the fact that stigma may impede participant recruitment, we plan to choose one general practitioner with rich experience in providing CBR services in the community as the key informant to lead participant recruitment.

Regarding front-line health/social workers, this study will adopt a snowball methodology: first, the key informant will be asked to recommend two health workers and two social workers who are directly involved in the provision of CBR services as interviewees. Then, each interviewee will be asked to name one potential participant (healthcare/social worker) within their professional networks. Approximately 28-33 participants will be interviewed.

\section{Data collection and analysis}

The key informant will help deliver a brief introduction of the study and this 30-60 min semistructured interview to the target participants. A written invitation will be sent to all participants after the introduction. A written consent will be obtained before an interview is carried out.

Applying the GTM principle, the interview will be conducted as progressive repetitive cycles. During the interview, the following themes will be covered:

- What are the status quo of the implementation of CBR services in urban China?

\section{Table 1 Sampling method for semistructured interviews}

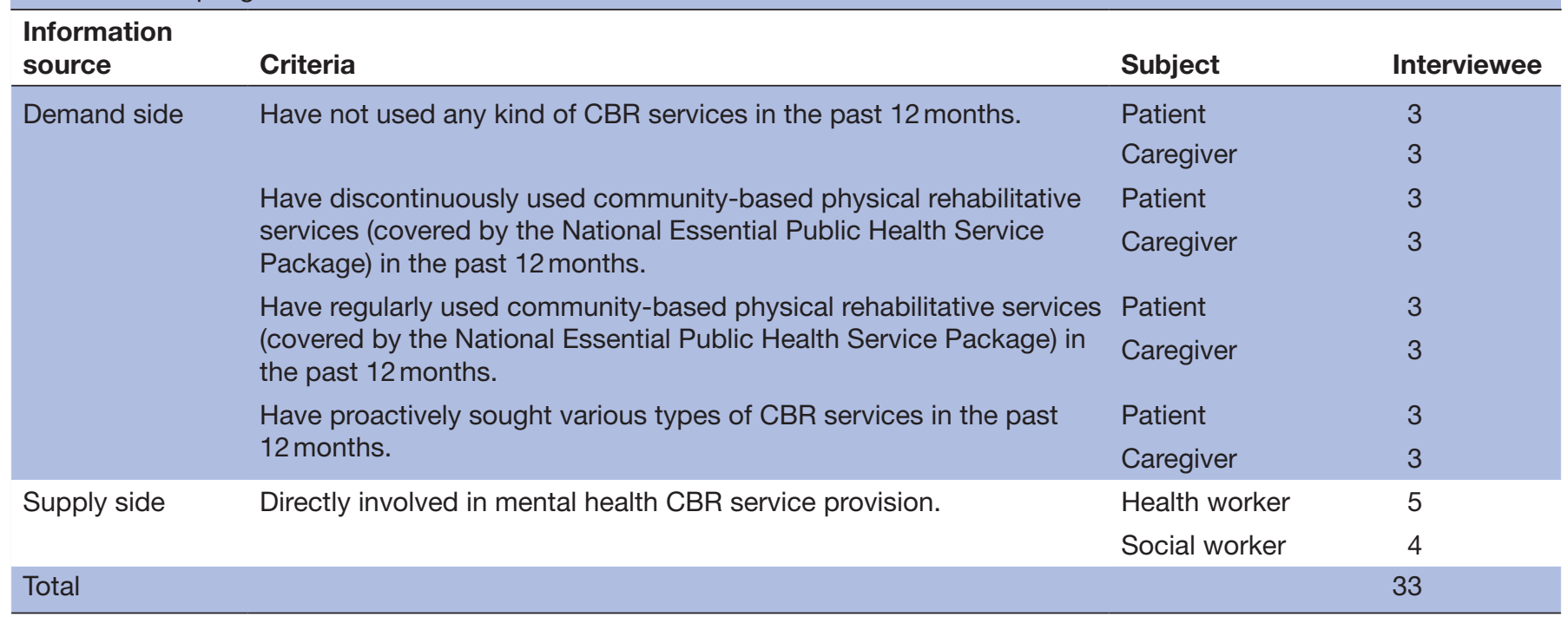

CBR, community-based rehabilitation. 
- Who makes decision on whether to use CBR services? How is the agreement achieved?

- What are the motivating factors patients with SMI or their caregivers have in terms of using CBR services? How are these sources of motivation developed?

- For patients with good prescription/medication adherence, what are their expected/perceived rewards? Is there a difference (and what is the difference) between what they had expected and what they have received?

- What are the perceived and practical obstacles that impede CBR service utilisation? How and to what extent did these factors influence tendency to use CBR services?

The recording will be transcribed verbatim and all data will be managed in NVivo V.10 software. The development of theme follows the principle of GTM, (Glaser BG, Strauss AL. The discovery of grounded theory: Strategies for qualitative research, Hawthorne, NY: Aldine 1967) including three steps: open coding, selective coding and theoretical coding. First, regarding each transcript, anything related to the aforementioned themes (normally rough information about phenomena) will be coded through line-by-line reading. At this stage, all transcripts will then be dissembled into small units, and the potential driving factors will be identified and coded. Second, the links (such as causal, associate or interactive relationships) between various driving factors will be preliminarily identified and factors with links will be merged into the same category. In the theoretical coding stage, relationships and links between different categories will be identified and three major clusters (motivation, rewards and costs) with factors from different layers will be extracted.

\section{Stage 2: form initial (theoretical) model using ISM}

In consideration of the complexity of the integrated model itself, the interpretive structural modelling (ISM), a sophisticated technique that transforms unclear, poorly articulated models into well-defined models, (Govindan K, Palaniappan M, Zhu Q et al. Analysis of third party reverse logistics provider using interpretive structural modelling. International Journal of Production Economics, 2012; 140(1): 204-211) will be employed to form the initial model.

Approximately 10 professionals from the mental health, health policy, CBR service provision fields and local governments will be invited to jointly set up the model according to Malone's procedure (Malone DW. An introduction to the application of interpretive structural modelling ISM. Proceedings of the IEEE, 1975; 63(3): 397-404) (figure 2). After professionals have reviewed the comprehensiveness of the factors identified in stage 1, this computer-assisted learning process will be employed to help experts develop relationships between any two factors, categorise and prioritise each factor into different layers, and therefore formulate the theoretical model that delineates how motivating

\begin{tabular}{|c|}
\hline $\begin{array}{l}\text { Step 1: Develop Structural Self-Interaction Matrix (SSIM) } \\
\text { 1. Review and organise potential factors identified in Stage } 1 \\
\text { 2. Specify and finalise the contextual relationship among the } \\
\text { interactions between any two factors ( } \mathrm{i} \text { and } \mathrm{j} \text { ) }\end{array}$ \\
\hline$\downarrow$ \\
\hline $\begin{array}{l}\text { Step 2: Develop Reachability Matrix } \\
\text { 1. Convert SSIM to Adjacency Matrix by assigning binary values } \\
\text { (1s or 0s) to the relationships } \\
\text { 2. Convert Adjacency Matrix to Reachability Matrix by adding } \\
1 * \text { entries to incorporate transitivity }\end{array}$ \\
\hline$\downarrow$ \\
\hline $\begin{array}{l}\text { Step 3: Stratify factors } \\
\text { 1. Extract reachability set and antecedent set for each factor } \\
\text { 2. Determine ISM hierarchy and club factors to form Conical } \\
\text { Matrix }\end{array}$ \\
\hline$\downarrow$ \\
\hline $\begin{array}{l}\text { Step 4: Develop diagraph } \\
\text { 1. Visualise Conical Matrix using nodes and lines of edges } \\
\text { 2. Develop final diagraph by removing indirect links }\end{array}$ \\
\hline
\end{tabular}

Figure 2 Flow diagram for developing the theoretical model. ISM, interpretive structural modelling. 
factors, costs and rewards influence one's tendency to use CBR services in China. Research hypotheses will be generated accordingly.

\section{Stage 3: statistically validate theoretical model using SEM}

In stage 3, a cross-sectional survey will be conducted to test the validity of the theoretical model developed in stage 2. SEM will be employed to test the loadings of each hypothesised pathway in order to validate the direction and magnitude of impact. This, on the one hand, will reveal how the factors from cultural, community, organisational and family levels influence family decision-makers' (either the patient or their family caregiver) tendency to use CBR services in China's context, and on the other hand help to identify any factor of intervention to facilitate evidence-based policy-making.

\section{Participant recruitment}

The study will employ a multistage sampling methodology to consider generalisability and feasibility issues.

The study will first select three cities (one in Western China, one in Eastern China and one in Central China) to enhance representativeness of findings, according to the following principles:

- The sample sites can represent the status quo of the geographical regions they are located.

- To investigate the impact of factors such as social culture and type of service on patients'/family's decision-making, there should be variations in social culture and type of CBR services among the sample sites.

The sample size was calculated based on the minimum sample size required for SEM: 10 cases per variable. (Nunnally JC. Psychometric theory. New York, NY: McGraw-Hill 1967) Approximately 25-30 variables would be determined, indicating the required minimum sample population of 300 individuals (patient or caregiver). Taking account of the prevalence of SMI (at least 0.5\%) (Baxter AJ, Charlson FJ, Cheng HG, et al. Prevalence of mental, neurological, and substance use disorders in China and India: A systematic analysis. Lancet Psychiatry, 2016; 3 (9): 832-841) and the response rate (set at $50 \%$ ), the expected population our sample sites cover was 120000 , which equals the coverage of 6 communities (one normally covers 20000 population).

Therefore, in each city, the study will select one community where the CHC provides the highest quality of physical CBR services (high level of patient adherence) as a research site. Then, we calculate its distance $(\mathrm{X} \mathrm{km})$ to the nearest facility that provides psychiatric rehabilitation services. All CHCs with X km's distance from the nearest psychiatric rehabilitation services within the city will be identified and one will be randomly selected. The community where this selected CHC is located will be selected as another research site. In the end, a total of six communities will be selected as our research sites. Attempts will be made to include all families (registered) with a member or more suffering from SMI who live in these six communities. One primary decision-maker (either the patient or their primary caregiver) of each registered family will be invited to complete the questionnaire.

\section{Questionnaire development}

The questionnaire is developed to test the validity/ explainability of the theoretical decision-making model. Regarding the measurable factors identified in the theoretical model, such as family burden and social support, the widely adopted Social Support Rating Scale and the Burden Scale for Family Caregivers will be included; regarding those latent variables that lack sophisticated scales to measure, a systematic review will be conducted and experts will be consulted to select appropriate observable variables.

The questionnaire includes information on the following:

- Patients' and their family primary caregivers' sociodemographic characteristics (age, gender, education, marital status, income, who makes the decision and so on).

- Utilisation of CBR services (intention, type, frequency, length and so on).

- Factors that may influence decision-maker's tendency (motivation, rewards and costs).

The items will be pilot-tested for face validity (content and structure) and test-retest reliability (1 week apart). Items with acceptable reliability (Cronbach's $\alpha \geq 70 \%$ ) will be included in the final survey instrument.

\section{Data collection and analysis}

The anonymised questionnaire will be distributed via a web link to Wenjuanxing (an online survey platform). Participants can complete the questionnaire using mobile phones or desktop. Reminders will be sent via text message or telephone 2 weeks after dissemination.

The analysis will be conducted in two phases:

Phase 1 will include descriptive analysis examining CBR service utilisation patterns and the variations among non-users, those who use CBR services occasionally and those who use CBR services regularly.

Phase 2 will use SEM to test the theoretical decision-making model using AMOS. Analysing procedure will follow Anderson and Gerbing's (Anderson JC, Gerbing DW. Structural equation modelling in practice: A review and recommended two-step approach. Psychological Bulletin, 1988; 103(3): 411-423) two-step modelling process, including measurement model and structural model. Regarding the measurement model, confirmatory factors analysis will be employed to examine the reliability of each construct using Cronbach's $\alpha$, composite reliability and the significance of factor loading for each item. The convergent validity and the discriminant validity will be assessed by average variance extracted (AVE), the comparison of the square root of AVEs and construct correlations, respectively. Regarding the structural model, the path coefficients of each relationship will be estimated and the goodness of fit will be assessed by 
indices such as $\mathrm{X}^{2}$ test, Normed Fit Index and Comparative Fit Index.

\section{Patient and public involvement}

Patient and public involvement was not sought in the design of this study protocol nor in the development of the research questions. Patient involvement in the recruitment and conduct of this study is described in the Participant recruitment and Data collection and analysis sections of this protocol. Participants will be asked during the recruitment stage if they want to be informed about the study findings. We will disseminate our findings (edited in lay language) to those who indicate interests in our findings via the key informant or email (if the participant is willing to provide his/her email address).

\section{ETHICS AND DISSEMINATION}

Rules on confidentiality and anonymity of data will be strictly followed. Prior to the interview or survey, all participants will be informed that they have the right to refuse to participate and withdraw from the study at any time without questions being asked. Information on participants' rights will be stated on the opening page of the survey. For participants who participate in the online survey, a completed questionnaire is deemed as having provided consent. Written consents will be obtained from participants before interviews are carried out. All interview and questionnaire data will be anonymised.

We will disseminate findings to the scientific community, policy-makers and healthcare providers via international and domestic peer-reviewed journals, conferences and various symposiums. The major findings of the project will be submitted to the National Natural Science Foundation of China. We aim to use the decision-making model to contribute towards the cumulative knowledge of underlying fundamental factors that affect the effective utilisation of mental health CBR services in LMICs. The identified factors of intervention and the verified effective services can be valuable in assisting community-level care providers to address gaps between need and provision.

\section{Author affiliations}

${ }^{1}$ School of Medicine and Health Management, Tongji Medical College, Huazhong University of Science and Technology, Wuhan, China

${ }^{2}$ School of Sociology and Social Policy, University of Nottingham, Nottingham, UK ${ }^{3}$ College of Public Health, University of Georgia, Athens, Georgia, USA

${ }^{4}$ Nanyou Community Health Service Center, Shenzhen Nanshan Center for Chronic Disease Control, Shenzhen, China

${ }^{5}$ School of Pharmacy, Tongji Medical College, Huazhong University of Science and Technology, Wuhan, China

Acknowledgements The authors would like to thank Dr Yongjie Zhou from the Affiliated Mental Health Center of Tongji Medical College, Huazhong University of Science and Technology for providing professional suggestions in developing the study protocol and questionnaire from a psychiatrist's perspective.

Contributors RW, DF, ST and ZF conceived the study. RW, DF, ST, YL, HS and QF contributed to the design of the study. RW, IS, ZC, TW, HF, YH and XC drafted the manuscript. All authors contributed to revising the manuscript.

Funding This study is supported by the National Natural Science Foundation of China (No 71704059, 71804052, 71801100) and the Fundamental Research Funds for the Central Universities (No 2017KFYXJJ156).
Competing interests None declared.

Patient consent Not required.

Ethics approval Ethical approval was granted by the Ethics Committee of Tongji Medical College, Huazhong University of Science and Technology (№ 2017S319).

Provenance and peer review Not commissioned; externally peer reviewed.

Open access This is an open access article distributed in accordance with the Creative Commons Attribution Non Commercial (CC BY-NC 4.0) license, which permits others to distribute, remix, adapt, build upon this work non-commercially, and license their derivative works on different terms, provided the original work is properly cited, appropriate credit is given, any changes made indicated, and the use is non-commercial. See: http://creativecommons.org/licenses/by-nc/4.0/.

\section{REFERENCES}

1. Steel Z, Marnane C, Iranpour C, et al. The global prevalence of common mental disorders: a systematic review and meta-analysis 1980-2013. Int J Epidemiol 2014;43:476-93.

2. Whiteford HA, Degenhardt L, Rehm J, et al. Global burden of disease attributable to mental and substance use disorders: findings from the global burden of disease study 2010. Lancet 2013;382:1575-86.

3. Foldemo A, Gullberg M, Ek AC, et al. Quality of life and burden in parents of outpatients with schizophrenia. Soc Psychiatry Psychiatr Epidemiol 2005;40:133-8.

4. Almeida OP, Hankey GJ, Yeap BB, et al. Mortality among people with severe mental disorders who reach old age: a longitudinal study of a community-representative sample of 37,892 men. PLoS One 2014;9:e111882.

5. World Health Organization. Mental health atlas. Geneva: World Health Organization, 2011.

6. World Health Organization. Mental health atlas 2014. Geneva: World Health Organization, 2015.

7. World Health Organization. Mental health action plan 2013-2020. Geneva: World Health Organization, 2013.

8. World Health Organization. mhGAP: Mental Health Gap Action Programme: Scaling up care for mental, neurological and substance use disorders. Geneva: World Health Organization, 2008.

9. Chisholm D, Flisher AJ, Lund C, et al. Scale up services for mental disorders: a call for action. Lancet 2007;370:1241-52.

10. Chatterjee S, Patel V, Chatterjee A, et al. Evaluation of a communitybased rehabilitation model for chronic schizophrenia in rural India. $\mathrm{Br}$ J Psychiatry 2003;182:57-62.

11. Jacob KS, Sharan P, Mirza J, et al. Global Mental Health 4 Mental health systems in countries: where are we now? Lancet 2007;370:1061-77.

12. Liu S, Page A. Reforming mental health in China and India. Lancet 2016;388:314-6.

13. Li K, Sun X, Zhang $Y$, et al. Mental health care in China: review on the delivery and policy issues in 1949-2009 and the outlook for the next decade. Acta Neuropsychiatr 2014;26:134-45.

14. State Council of People's Republic of China. National mental health work plan (2015-2020). Beijing: General Office of the State Council, 2015.

15. National Health and Family Planning Commission. Work specification on management and treatment of severe mental illness. Beijing: National Health and Family Planning Commission, 2012.

16. Ministry of Health, Publicity Department of the CPC Central Committee, National Development and Reform Commission, et al. National mental healthcare system guidelines. Beijing: Chinese Center for Disease Control and Prevention, 2008.

17. Standing Committee of the National People's Congress. National Mental Health Law Beijing: Standing Committee of the National People's Congress. 2012.

18. Zheng H, Zhou J, Fu Z. Study on service situation and development strategies of community mental health services for schizophrenia. Chinese Primary Health Care 2012;26:7-9.

19. Yang Q. Study on community rehabilitation management of patients with mental disorders. Chinese: Nanchang University, 2013.

20. Li H. Study on management of community-based rehabilitation model for mental disabled people - taking shanghai sunshine soul park as example. Chinese: Fudan University, 2013.

21. Jacob KS. Community care for people with mental disorders in developing countries: problems and possible solutions. $\mathrm{Br} \mathrm{J}$ Psychiatry 2001;178:296-8.

22. Kang H, Guan L, Wang X, et al. Economic condition basic medical insurance and medication among patients with severe mental illness in 31 demonstration cities for '686 program' implementation. Chinese Journal of Public Health 2015;31:997-1001. 
23. Li L. Community mental healthcare system awaits further development. China Hospital CEO 2012(8):62-3.

24. Phillips MR. Can China's new mental health law substantially reduce the burden of illness attributable to mental disorders? Lancet 2013;381:1964-6.

25. Prince M, Patel V, Saxena S, et al. No health without mental health. Lancet 2007:370:859-77.

26. Wang PS, Aguilar-Gaxiola S, Alonso J, et al. Use of mental health services for anxiety, mood, and substance disorders in 17 countries in the WHO world mental health surveys. Lancet 2007;370:841-.

27. Wong YJ, Tran KK, Kim SH, et al. Asian Americans' lay beliefs about depression and professional help seeking. J Clin Psychol 2010;66:n/ a-32.

28. Andrade LH, Alonso J, Mneimneh Z, et al. Barriers to mental health treatment: results from the WHO World Mental Health surveys. Psychol Med 2014;44:1303-17.

29. Coleman SJ, Stevelink SAM, Hatch SL, et al. Stigma-related barriers and facilitators to help seeking for mental health issues in the armed forces: a systematic review and thematic synthesis of qualitative literature. Psychol Med 2017;47:1880-92.

30. Lannin DG, Vogel DL, Brenner RE, et al. Does self-stigma reduce the probability of seeking mental health information? J Couns Psychol 2016;63:351-8

31. Jorm AF. Mental health literacy: empowering the community to take action for better mental health. Am Psychol 2012;67:231-43.

32. Gulliver A, Griffiths KM, Christensen $\mathrm{H}$. Perceived barriers and facilitators to mental health help-seeking in young people: a systematic review. BMC Psychiatry 2010;10:113.

33. Mohamed S, Rosenheck R, He H, et al. Insight and attitudes towards medication among inpatients with chronic schizophrenia in the US and China. Soc Psychiatry Psychiatr Epidemiol 2014;49:1063-70.

34. Patel V, Xiao S, Chen $\mathrm{H}$, et al. The magnitude of and health system responses to the mental health treatment gap in adults in India and China. Lancet 2016;388:3074-84.

35. Tsai YF, Ku YC. Self-care symptom management strategies for auditory hallucinations among inpatients with schizophrenia at a veterans' hospital in Taiwan. Arch Psychiatr Nurs 2005;19:194-9.

36. Mackenzie CS, Gekoski WL, Knox VJ. Age, gender, and the underutilization of mental health services: the influence of helpseeking attitudes. Aging Ment Health 2006;10:574-82.

37. Mo PK, Mak WW. Help-seeking for mental health problems among Chinese: the application and extension of the theory of planned behavior. Soc Psychiatry Psychiatr Epidemiol 2009;44:675-84.

38. Boydell KM, Gladstone BM, Volpe T. Understanding help seeking delay in the prodrome to first episode psychosis: a secondary analysis of the perspectives of young people. Psychiatr Rehabil $J$ 2006;30:54-60.

39. Vogel DL, Wade NG, Wester SR, et al. Seeking help from a mental health professional: the influence of one's social network. J Clin Psychol 2007;63:233-45.

40. Evans-Lacko S, Brohan E, Mojtabai R, et al. Association between public views of mental illness and self-stigma among individuals with mental illness in 14 European countries. Psychol Med 2012:42:1741-52

41. Corrigan PW, Morris SB, Michaels PJ, et al. Challenging the public stigma of mental illness: a meta-analysis of outcome studies. Psychiatr Serv 2012;63:963-73.

42. Tieu Y, Konnert CA. Mental health help-seeking attitudes, utilization, and intentions among older Chinese immigrants in Canada. Aging Ment Health 2014;18:140-7.

43. Chen $\mathrm{H}$, Fang $\mathrm{X}$, Liu C, et al. Associations among the number of mental health problems, stigma, and seeking help from psychological services: a path analysis model among Chinese adolescents. Child Youth Serv Rev 2014;44:356-62.

44. Chin WY, Chan KT, Lam CL, et al. Help-seeking intentions and subsequent 12-month mental health service use in Chinese primary care patients with depressive symptoms. BMJ Open 2015;5:e006730.

45. Razzouk D, Sharan P, Gallo C, et al. Scarcity and inequity of mental health research resources in low-and-middle income countries: a global survey. Health Policy 2010;94:211-20.

46. Baingana F, al'Absi M, Becker AE, et al. Global research challenges and opportunities for mental health and substance-use disorders. Nature 2015;527:S172-S177.

47. Yu Y, Liu ZW, Hu M, et al. Mental Health Help-Seeking Intentions and Preferences of Rural Chinese Adults. PLoS One 2015;10:e0141889.

48. Wong DFK, Li JCM. cultural influence on shanghai Chinese people's help-seeking for mental health problems: implications for social work practice. Br J Soc Work 2014;44:868-85.

49. Kantor V, Knefel M, Lueger-Schuster B. Perceived barriers and facilitators of mental health service utilization in adult trauma survivors: a systematic review. Clin Psychol Rev 2017;52:52-68.

50. Tang $Y$, Zheng H. Evaluation of patients' families on social workers involved in the community services of severe psychoses in Shanghai. Med Soc (Berkeley) 2016;29:47-9.

51. Young JT. Illness behaviour: a selective review and synthesis. Sociol Health IIIn 2004;26:1-31.

52. O'Connor PJ, Martin B, Weeks CS, et al. Factors that influence young people's mental health help-seeking behaviour: a study based on the health belief model. J Adv Nurs 2014;70:2577-87.

53. Andersen R, Aday LA. Access to medical care in the U.S.: realized and potential. Med Care 1978;16:533-46.

54. Andersen RM. National health surveys and the behavioral model of health services use. Med Care 2008;46:647-53.

55. Bradley EH, McGraw SA, Curry L, et al. Expanding the andersen model: the role of psychosocial factors in long-term care use. Health Serv Res 2002;37:1221-42.

56. Roh S, Burnette $\mathrm{CE}$, Lee $\mathrm{KH}$, et al. Predicting help-seeking attitudes toward mental health services among american indian older adults: is andersen's behavioral model a good fit? J App/ Gerontol 2017;36:94.

57. Jang Y, Chiriboga DA, Okazaki S. Attitudes toward mental health services: age-group differences in korean american adults. Aging Ment Health 2009;13:127-34.

58. Henshaw EJ, Freedman-Doan CR. Conceptualizing mental health care utilization using the health belief model. Clinical Psychology: Science and Practice 2009;16:420-39.

59. Castonguay J, Filer CR, Pitts MJ. Seeking help for depression: applying the health belief model to illness narratives. South Commun J 2016;81:289-303.

60. Homans GC. Social Behavior as Exchange. Am J Sociol 1958:63:597-606.

61. Hamrin V, McCarthy EM, Tyson V. Pediatric psychotropic medication initiation and adherence: a literature review based on social exchange theory. J Child Adolesc Psychiatr Nurs 2010;23:151-72

62. Prizer LP, Gay JL, Perkins MM, et al. Using social exchange theory to understand non-terminal palliative care referral practices for parkinson's disease patients. Palliat Med 2017;31:861-7.

63. Aryee S, Walumbwa FO, Mondejar R, et al. Accounting for the influence of overall justice on job performance: integrating selfdetermination and social exchange theories. Journal of Management Studies 2015;52:231-52.

64. Guillet E, Sarrazin P, Carpenter PJ, et al. Predicting persistence or withdrawal in female handballers with social exchange theory. International Journal of Psychology 2002;37:92-104. 